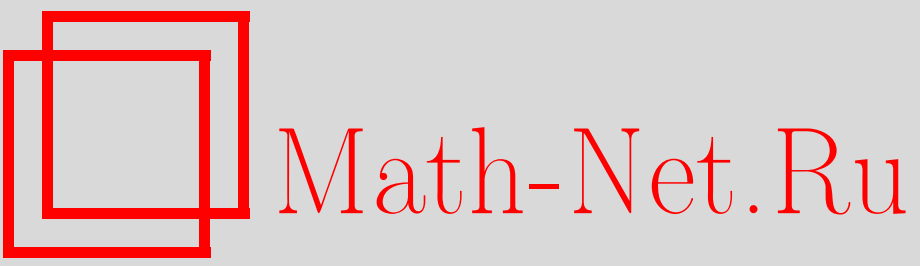

Г. П. Джорджадзе, Г. Вайгт, Проблема нулевых мод в теории поля Лиувилля, TMФ, 2004, том 139, номер 2, 245-267

DOI: https://doi.org/10.4213/tmf52

Использование Общероссийского математического портала Math-Net.Ru подразумевает, что вы прочитали и согласны с пользовательским соглашением

http://www.mathnet.ru/rus/agreement

Параметры загрузки:

IP: 52.205 .19 .152

26 апреля 2023 г., $16: 45: 13$ 
ТЕОРЕТИЧЕСКАЯ

И МАТЕМАТИЧЕСКАЯ

ФИЗИКА

Том 139, № 2

май, 2004

(C) 2004 г.

Г. Джорджадзе*, Г. Вайгт ${ }^{\dagger}$

\section{ПРОБЛЕМА НУЛЕВЫХ МОД В ТЕОРИИ ПОЛЯ ЛИУВИЛЛЯ}

Произведено квантование нулевых мод свободного поля $p, q$ на полуплоскости $p>0$ как для теории поля Лиувилля, так и для ее редукции к динамике лиувиллевской частицы. Детально описана динамика лиувиллевской частицы, вычислены одноточечные функции для вершинных операторов частицы, выведена их реализация посредством нулевых мод на полуплоскости и доказано, что вершинные операторы частицы являются самосопряженными в гильбертовом пространстве $L^{2}\left(\mathbb{R}_{+}\right)$вследствие симметрий, порождаемых $S$-матрицей. Аналогично самосопряженность соответствующего вершинного оператора теории поля Лиувилля в секторе нулевых мод получена с применением лиувиллевской амплитуды отражения, которая выводится при помощи операторного метода.

Ключевые слова: конформная теория поля, теория Лиувилля, гамильтонова редукция, динамика лиувиллевской частицы, нулевые моды, квантование на полуплоскости.

\section{1. ВВЕДЕНИЕ}

Несмотря на то что теория поля Лиувилля интенсивно изучалась в различных областях математики и физики, ее квантово-механическая картина остается неполной в отношении некоторых фундаментальных вопросов. Однако достаточно простой вид общего решения [1], его свободно-полевые представления [2]-[6] и отдельные полученные точные квантовые результаты [4]-[7] дают большие надежды на построение полного описания. Рассматриваемая нами нерешенная проблема - это проблема квантования канонических нулевых мод свободного поля $p, q$ на полуплоскости $p>0$ [4], [7], которые описывают вакуум регулярной периодической теории поля Лиувилля. Эта проблема возникает вследствие особенности лиувиллевской динамики.

В настояшей работе мы квантуем эти канонические нулевые моды и, в частности, доказываем самосопряженность вершинных операторов в гильбертовом пространстве $L^{2}\left(\mathbb{R}_{+}\right)$путем рассмотрения симметрий, порождаемых $S$-матрицей. Мы предполагаем довести до конца нашу предыдущую работу по периодической теории Лиувилля [7], для

* Математический институт им. А. М. Размадзе, Тбилиси, Грузия. E-mail: jorj@rmi.acnet.ge

${ }^{\dagger}$ DESY Zeuthen, Zeuthen, Germany. E-mail: weigt@ifh.de 
того чтобы получить возможность вычислять корреляционные функции. Более того, решение этой проблемы нулевых мод позволило бы также далее развить квантование $S L(2, \mathbb{R}) / U(1)$-модели черной дыры, рассмотренной в работе [8]. В действительности такие исследования являются фундаментальными для квантования калибровочных теорий Весса-Зумино-Новикова-Виттена (ВЗНВ). Наши вычисления основаны на точном каноническом операторном формализме в гильбертовом пространстве. Однако в этой работе мы не следуем высказанному нами ранее [7] предложению использовать формализм когерентных состояний, которьй учитывает трансляционную симметрию и симметрию преобразования подобия полуплоскости.

Нулевые моды исследуются с помощью канонического отображения из плоскости в полуплоскость. Такое отображение естественно возникает для динамики частицы в экспоненциальном потенциале при переходе от координат фазового пространства частицы к их асимптотическим ( in- или out-) переменным. Такой, основанньй на динамике частицы, подход кажется полезным, поскольку теорию поля Лиувилля можно редуцировать к механической модели, а относительно динамики лиувиллевской частицы многое уже известно [3], [9]-[11]. Однако квантование на полуплоскости до сих пор не было рассмотрено в достаточной мере. В частности, не установлена самосопряженность действия $q$-экспонент в пространстве $L^{2}\left(\mathbb{R}_{+}\right)$, и поэтому использование лиувиллевских вершинных операторов для вычисления лиувиллевских корреляционных функций до сих пор не полностью оправданно. Подробное рассмотрение динамики лиувиллевской частицы окажется полезным для понимания структуры нулевых мод теории поля Лиувилля.

В разделе 2 дается краткое описание свободно-полевого представления периодической теории Лиувилля и формулируется проблема нулевых мод. Там же описывается классическая и квантово-механическая редукции теории Лиувилля к динамике частицы и приводится каноническое преобразование к нулевым модам свободного поля на полуплоскости. В разделе 3 детально исследуется квантовая динамика частицы в экспоненциальном потенциале, с использованием матрицы Мёллера строится квантовая реализация соответствующих операторов нулевых мод, вычисляются матричные элементы механического лиувиллевского вершинного оператора и из них выводится вершинньй оператор частишы, выраженный в терминах нулевых мод полуплоскости. Здесь необходимо осуществить продолжение матричных элементов, которые должны рассматриваться как обобщенные функции, по параметру взаимодействия. Самосопряженное действие вершинных операторов в гильбертовом пространстве $L^{2}\left(\mathbb{R}_{+}\right)$может быть реализовано как в случае частицы, так и в секторе нулевых мод теории поля с использованием симметрий вершин при отражении. Амплитуда отражения в теории поля Лиувилля выводится с помощью операторного метода. Удивительно, что эта амплитуда оказывается тождественной амплитуде, которая была получена в работе [12] с помощью симметрии трехточечной корреляционной функции, предложенной в [13] на основе континуального интегрирования. В разделе 4 мы суммируем результаты и подводим итоги. Некоторые детали вынесены в приложения. 


\section{2. НУЛЕВЫЕ МОДЫ ПЕРИОДИЧЕСКОЙ ТЕОРИИ ЛИУВИЛЛЯ}

Для того чтобы получить удобную формулировку проблемы нулевых мод, мы опишем каноническую свободно-полевую параметризацию периодической теории Лиувилля, сведем ее к динамике лиувиллевской частицы (в классическом и квантово-механическом случаях) и увидим, что координаты фазового пространства частищы асимптотически связаны с нулевыми модами свободного поля на полуплоскости посредством некоторого канонического преобразования, которое является классическим аналогом матрицы Мёллера.

2.1. Периодическая теория поля Лиувилля. Решения уравнения Лиувилля

$$
\varphi_{\tau \tau}(\tau, \sigma)-\varphi_{\sigma \sigma}(\tau, \sigma)+\frac{4 m^{2}}{\gamma} e^{2 \gamma \varphi(\tau, \sigma)}=0
$$

имеют удобное свободно-полевое представление [2], [3]

$$
e^{-\gamma \varphi(\tau, \sigma)}=e^{-\gamma \phi(\tau, \sigma)}\left(1+m^{2} \iint d y d \bar{y} G(z, \bar{z} ; y, \bar{y}) e^{2 \gamma \phi(y)} e^{2 \gamma \phi(\bar{y})}\right)
$$

где $\gamma$ и $m$ - положительные константы, $z=\tau+\sigma$ и $\bar{z}=\tau-\sigma-$ координаты светового конуса, $\phi(z), \bar{\phi}(\bar{z})$ - киральная и антикиральная компоненты свободного поля $\phi(\tau, \sigma)$, $\phi(\tau, \sigma)=\phi(z)+\bar{\phi}(\bar{z}), \quad G(z, \bar{z} ; y, \bar{y})-$ функция Грина оператора Даламбера $\partial_{z \bar{z}}^{2}$. Для периодических граничных условий $\varphi(\tau, \sigma+2 \pi)=\varphi(\tau, \sigma)$ область интегрирования в $(2)$ есть $(y, \bar{y}) \in[0,2 \pi] \times[0,2 \pi]$, параметризирующее свободное поле $\phi(\tau, \sigma)$ также является периодическим и может быть разложено в ряд Фурье:

$$
\phi(\tau, \sigma)=q+\frac{p}{2 \pi} \tau+\frac{i}{\sqrt{4 \pi}} \sum_{n \neq 0} \frac{1}{n}\left(a_{n} e^{-i n(\tau+\sigma)}+\bar{a}_{n} e^{-i n(\tau-\sigma)}\right) .
$$

Канонические скобки Пуассона для нулевых мод и осцилляторов

$$
\{p, q\}=1, \quad\left\{a_{m}, a_{n}\right\}=i m \delta_{m+n, 0}=\left\{\bar{a}_{m}, \bar{a}_{n}\right\}
$$

определяют соотношение (2) как каноническое преобразование между полем Лиувилля и свободным полем.

Для периодических граничных условий функция Грина $G$ в формуле $(2)$ может быть записана в виде [6]

$$
G(z, \bar{z} ; y, \bar{y})=\theta_{\gamma p}(z-y) \theta_{\gamma p}(\bar{z}-\bar{y})
$$

где

$$
\theta_{\gamma p}(z-y)=\frac{e^{\gamma p \epsilon(z-y) / 2}}{2 \operatorname{sh}(\gamma p / 2)}
$$


- функция Грина, обрашаюшая оператор $\partial_{z}$ на функциях $A(z)$ с монодромией $A(z+$ $2 \pi)=e^{\gamma p} A(z), \quad p \neq 0$. Функция $\epsilon(z)-$ ступенчатая функция, дифференцирование которой дает периодическую $\delta$-функцию. Точка $p=0$ является сингулярной и будет исключена из (6). Можно показать, что значения $p>0$ и $p<0$ покрьвают класс всех регулярных периодических лиувиллевских полей, и для того чтобы избежать двойного счета решений (2), предполагается, что нулевые моды лежат только в полуплоскости $p>0$. Это динамическое ограничение требует, однако, дополнительных исследований при квантовании [7]. Гильбертово пространство ненулевых мод $a_{n}, \bar{a}_{n}$ является обычным фоковским пространством, тогда как гильбертово пространство нулевых мод свободного поля определяется волновыми функциями $\psi(p) \in L^{2}\left(\mathbb{R}_{+}\right)$. K сожалению, обычный оператор координаты в импульсном представлении $\hat{q}=i \hbar \partial_{p}$ не самосопряжен в пространстве $L^{2}\left(\mathbb{R}_{+}\right)$, и для использования появляющихся в (2) операторных экспонент $e^{ \pm \gamma q}$ последние должны быть надлежащим образом определены. Эта проблема эрмитовости связана с отсутствием трансляционной симметрии на полуплоскости $p>0$ в $p$-направлении. Однако мы покажем, что $S$-матричные преобразования позволяют "восстановить" эту симметрию заменой $L^{2}\left(\mathbb{R}_{+}\right) \rightarrow L^{2}\left(\mathbb{R}^{1}\right)$, где самосопряженность операторов $e^{ \pm \gamma \hat{q}}$ очевидна.

2.2. Редукции теории Лиувилля к модели частицы. Периодическую теорию Лиувилля можно редуцировать к механической модели Лиувилля различными способами. Поскольку лиувиллевские вакуумные конфигурации описываются однородным полем, $\partial_{\sigma} \varphi(\tau, \sigma)=0$, соответствуюшее поле Лиувилля зависит только от временно́й координаты $\varphi(\tau, \sigma)=Q(\tau)$ и уравнение Лиувилля (1) сводится к механической модели Лиувилля:

$$
\ddot{Q}(\tau)+\frac{4 m^{2}}{\gamma} e^{2 \gamma Q(\tau)}=0 .
$$

Редуцированный гамильтониан принимает вид

$$
H=\frac{1}{4 \pi}\left(P^{2}+4 \omega^{2} e^{2 \gamma Q}\right)
$$

где $P=2 \pi \dot{Q}$ - канонически-сопряженный импульс, параметр $\omega=2 \pi m / \gamma$. Киральный тензор энергии-импульса

$$
T(z)=\left(\partial_{z} \varphi\right)^{2}-\frac{1}{\gamma} \partial_{z z}^{2} \varphi
$$

в таком случае является положительной константой $T(z)=c^{2}$, и его свободно-полевое представление

$$
T(z)=\phi^{\prime 2}(z)-\frac{1}{\gamma} \phi^{\prime \prime}(z)
$$

приводит к равенству $\phi^{\prime}(z)=c$, которое требует обращения в нуль осцилляторных мод, $a_{n}=0=\bar{a}_{n}$. Как следствие решение уравнения Лиувилля (2)-(6) сводится к обшему 
решению уравнения для частицы (7)

$$
e^{-\gamma Q(\tau)}=e^{-\gamma(q+p \tau /(2 \pi))}+\frac{\omega^{2}}{p^{2}} e^{\gamma(q+p \tau /(2 \pi))} .
$$

Очевидно, что механическая модель Лиувилля может быть также получена из теории Лиувилля путем гамильтоновой редукции со связями второго рода $a_{n}=0=\bar{a}_{n}$ и что редуцированный гамильтониан (8) канонически связан посредством (9) со свободным гамильтонианом вида

$$
H=\frac{p^{2}}{4 \pi} .
$$

Стоит отметить, что приведенный выше результат может быть получен прямо из $S L(2, \mathbb{R})$-теории ВЗНВ [14], [7] или из ее однородной разновидности, $S L(2, \mathbb{R})$-модели частицы [11], с помошью подходящей гамильтоновой редукции. Если отождествить калибровочно-инвариантную часть $S L(2, \mathbb{R})$-поля, обозначенную в $[7]$ как $g_{12}(\tau)$, с вершинной функцией частицы $V(\tau)=e^{-\gamma Q(\tau)}$, то из $S L(2, \mathbb{R})$-уравнений движения частицы

$$
\ddot{g}_{\alpha \beta}(\tau)=\frac{\gamma^{2}}{\pi} H g_{\alpha \beta}(\tau)
$$

получим калибровочно-инвариантное уравнение

$$
\ddot{V}(\tau)=\frac{\gamma^{2}}{\pi} H V(\tau),
$$

эквивалентное (7). Интегрирование последнего уравнения с использованием (10) в виде

$$
p=\sqrt{4 \pi H}
$$

снова дает обшее решение (9).

Интересно обсудить остаток конформной симметрии

$$
\varphi(z, \bar{z}) \rightarrow \varphi(f(z), \bar{f}(\bar{z}))+\frac{1}{2 \gamma} \ln f^{\prime}(z) \bar{f}^{\prime}(\bar{z})
$$

теории Лиувилля. Для $\sigma$-независимых лиувиллевских полей функции $f(z)$ и $\bar{f}(\bar{z})$ могут быть только линейными, и при параметризации $f(z)=a z+b, \bar{f}(z)=a \bar{z}+b$ преобразование (14) сводится к преобразованиям

$$
Q(\tau) \rightarrow Q(\tau+b), \quad Q(\tau) \rightarrow Q(a \tau)+\frac{\ln a}{\gamma}, \quad a>0 .
$$

Эти преобразования являются симметриями динамических уравнений (7) и (12), и они преобразуют нулевые моды $p, q$ решения (9) следуюшим образом:

$$
(p, q) \rightarrow\left(p, q+\frac{p b}{2 \pi}\right), \quad(p, q) \rightarrow\left(a p, q+\frac{\ln a}{\gamma}\right) .
$$

С двиги по времени, очевидно, порождаются свободным гамильтонианом (10), в то время как оставшиеся преобразования подобия не являются каноническими, поскольку они преобразуют действие как $S \rightarrow a S$ и данная симметрия не является нётеровской. 
2.3. Асимптотики механической модели Лиувилля. Механическая модель Лиувилля асимптотически становится свободной теорией. Общее решение (9) дает

$$
\lim _{\tau \rightarrow-\infty}[Q(\tau)-q(\tau)]=0, \quad \lim _{\tau \rightarrow-\infty}[P(\tau)-p]=0,
$$

где $q(\tau)=q+p \tau /(2 \pi)$ - решение для свободной частицы. Поэтому $p$ и $q$ могут интерпретироваться как $i n$-переменные динамики лиувиллевской частицы. Аналогично, рассматривая поведение решения (9) при $\tau \rightarrow+\infty$, введем out-переменные. Эти $i n-$ и out-переменные связаны преобразованием

$$
P_{\text {out }}=-p, \quad Q_{\text {out }}=-q+\frac{2}{\gamma} \ln \frac{p}{\omega},
$$

которое объединяет отражение $p, q$ с каноническим отображением. Важное наблюдение заключается в том, что общее решение (9) инвариантно относительно преобразования (18). В квантово-механическом случае эта симметрия будет порождаться $S$-матрицей теории частицы. Отметим, что в теории поля Лиувилля $i n$ - и out-поля [2] связаны специальным преобразованием Мёбиуса $A \rightarrow-\left(m^{2} A\right)^{-1}, \bar{A} \rightarrow-\left(m^{2} \bar{A}\right)^{-1}$, которое оставляет инвариантным обшее решение

$$
\varphi(\tau, \sigma)=\ln \frac{A^{\prime}(\tau+\sigma) \bar{A}^{\prime}(\tau-\sigma)}{\left[1+m^{2} A(\tau+\sigma) \bar{A}(\tau-\sigma)\right]^{2}} .
$$

Вследствие (9) координаты фазового пространства $P, Q$ при $\tau=0$ и асимптотические переменные $p, q$ связаны соотношениями

$$
e^{-\gamma Q}=e^{-\gamma q}+\frac{\omega^{2}}{p^{2}} e^{\gamma q}, \quad P=-p \operatorname{th}\left(\gamma q+\ln \frac{\omega}{p}\right) .
$$

Это каноническое и обратимое отображение плоскости $(P, Q)$ на всю полуплоскость $(p, q)$. Его обрашение дает (13) и делает $q$ функцией от $P, Q$. Для координаты $\tilde{q}=q+$ $(1 / \gamma) \ln (\omega / p)$ преобразование упрошается:

$$
\begin{array}{ll}
e^{-\gamma Q}=\frac{2 \omega}{p} \operatorname{ch} \gamma \tilde{q}, & 2 \omega \operatorname{sh} \gamma \tilde{q}=-P e^{-\gamma Q}, \\
P=-p \operatorname{th} \gamma \tilde{q}, & p=\sqrt{P^{2}+4 \omega^{2} e^{2 \gamma Q}},
\end{array}
$$

и может быть в итоге представлено с помощью производящей функции [10]

$$
F(Q, \tilde{q})=\frac{2 \omega}{\gamma} e^{\gamma Q} \operatorname{sh} \gamma \tilde{q} .
$$

По сушеству, проблема полуплоскости становится видна уже на $(P, Q)$-плоскости,где гамильтониан (8) непрерывно связьвает положительные $i n$ - и отрицательные out-импульсы, что показано на рис. 1. Каждая траектория в фазовом пространстве полной 


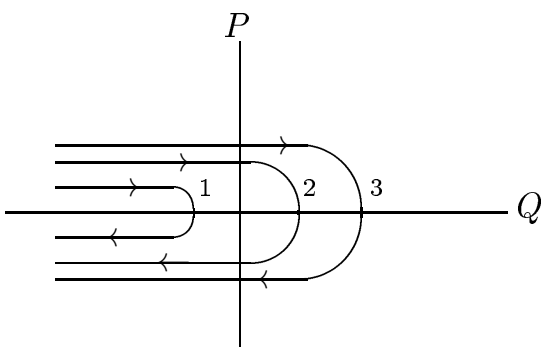

Рис. 1



Рис. 2

$(P, Q)$-плоскости взаимно однозначно отображается преобразованием $(21)$ в соответствующую прямую $p=$ const в верхней полуплоскости $(p, q)$ на рис. 2. Мы наблюдаем "невырожденность" на фазовом пространстве $(P, Q)$, которая в квантово-механическом случае будет характеризоваться невырожденностью спектра гамильтониана.

К сожалению, отображение (20) является нелинейным, и его квантовая реализация нетривиальна. Однако существует некоторая предельная процедура, которая упрощает вычисления в квантовом случае. В этой связи введем дополнительный свободный гамильтониан $H_{0}=P^{2} /(4 \pi)$ и рассмотрим гамильтоновы потоки $U_{H_{0}}(\tau)$ и $U_{H}(\tau)$, порождаемые соответственно $H_{0}$ и $H$ :

$$
U_{H_{0}}(\tau)(P, Q)=\left(P, Q+\frac{P \tau}{2 \pi}\right), \quad U_{H}(\tau)(P, Q)=(P(\tau), Q(\tau))
$$

Здесь $P(\tau), Q(\tau)$ описывают решение уравнений Гамильтона с начальными данными $P, Q$. Для композиции этих двух потоков $U(\tau)=U_{H}(\tau) \cdot U_{H_{0}}(-\tau)$ имеем

$$
U(\tau)(P, Q)=\left(P(\tau), Q(\tau)-\frac{P(\tau) \tau}{2 \pi}\right)
$$

и в силу (17) получаем каноническое отображение плоскости $(P, Q)$ на всю полуплоскость $(p, q)$ с помошью асимптотического соотношения

$$
\lim _{\tau \rightarrow-\infty} U(\tau)(P, Q)=(p, q)
$$

В квантово-механическом случае роль связующего звена в этом отображении играет матрица Мёллера. Отметим, что $U(\tau)$ определяет каноническое преобразование координат $P, Q$ для любого $\tau$.

Следует упомянуть, что формулы (5), (6) дают $G(z, \bar{z} ; y, \bar{y}) \rightarrow 0$ при $\tau \rightarrow-\infty$, и поэтому параметризирующее свободное поле (3) является асимптотическим $i n$-полем теории Лиувилля. Решение уравнения Лиувилля (2), как в случае частицы, может быть интерпретировано как сумма in- и out-полей, и сектор лиувиллевских нулевых мод задается $i n$-переменными механической модели. 
2.4. Редукция квантовой теории поля Лиувилля. Мы опишем редукцию квантовой теории поля Лиувилля к динамике квантовой частишы только в обших чертах.

Представление свободного поля (2)-(6) было использовано в [6] для алгебраического построения лиувиллевского вершинного оператора $\left(e^{\lambda \varphi(\tau, \sigma)}\right)$ op. С использованием определения операторного поля Лиувилля $\hat{\varphi}(\tau, \sigma)=\left.\partial_{\lambda}\left(e^{\lambda \varphi(\tau, \sigma)}\right)_{\mathrm{op}}\right|_{\lambda=0}$ было показано, что операторное уравнение Лиувилля и канонические перестановочные соотношения остаются справедливы, однако свободно-полевое описание лиувиллевских операторов деформируется по сравнению с классическим аналогом. Как осцилляторные операторы, так и операторы нулевых мод вносят совместный вклад в эти деформации и дают конформно-инвариантные, локальные и свободные от аномалий операторные структуры. Эти деформации также гарантируют, например, что четырехточечная функция 25-мерной некритической струны согласуется с критической амплитудой ШапироВирасоро в 26-мерном пространстве-времени Минковского.

Механическая модель Лиувилля получается гамильтоновой редукцией теории поля Лиувилля со связями $a_{n}=0=\bar{a}_{n}$. Такая редукция может быть воспроизведена в квантово-механическом случае вычислением осцилляторных вакуумных матричных элементов вершинных операторов

$$
\widehat{\mathbf{V}}_{\lambda}(\tau)=\left\langle\mathbf{0}\left|\left(e^{\lambda \varphi(\tau, \sigma)}\right)_{\mathrm{op}}\right| \mathbf{0}\right\rangle,
$$

где вакуумное состояние $|\mathbf{0}\rangle$ обрашается в нуль под действием осцилляторов $\hat{a}_{n}, \hat{\bar{a}}_{n}$ при $n>0$.

Этот матричный элемент, очевидно, является оператором в секторе нулевых мод, и можно доказать его $\sigma$-независимость. Тогда операторное уравнение Лиувилля просто сводится к операторному уравнению для частицы. Здесь следует, однако, указать, что с помошью этой редукции нам не удалось распутать вклады осцилляторов и нулевых мод, поскольку связи $a_{n}=0=\bar{a}_{n}$ суть связи второго рода. Тем не менее результат окажется полезным.

Для применения нам будет достаточно рассмотреть специальный лиувиллевский вершинный оператор только для $\lambda=-\gamma$. В приложении А этот оператор получен в виде суммы in-приходящего и деформированного out-уходящего членов:

$$
\begin{aligned}
\widehat{\mathbf{V}}(\tau)= & e^{-\gamma \hat{p} \tau /(4 \pi)} e^{-\gamma \hat{q}} e^{-\gamma \hat{p} \tau /(4 \pi)}+ \\
& +\omega_{\alpha}^{2} \frac{e^{\gamma \hat{p} \tau /(4 \pi)}}{\hat{p}} \frac{\Gamma(-i \gamma p /(2 \pi))}{\Gamma(i \gamma p /(2 \pi))} e^{\gamma \hat{q}} \frac{\Gamma(i \gamma p /(2 \pi))}{\Gamma(-i \gamma p /(2 \pi))} \frac{e^{\gamma \hat{p} \tau /(4 \pi)}}{\hat{p}},
\end{aligned}
$$

где

$$
\omega_{\alpha}=\frac{2 \pi m_{\alpha} \Gamma(1+2 \alpha)}{\gamma}
$$

а $m_{\alpha}$ задается в (П.3). Появление деформированного параметра $\omega_{\alpha}$ и Г-функций (П.6) обусловлено вкладами осцилляторов, а также нулевых мод, и оператор (26) имеет, конечно, классический предел (9). 


\section{3. КВАНТОВАНИЕ И САМОСОПРЯЖЕННЫЕ ВЕРШИННЫЕ ОПЕРАТОРЫ}

В этом разделе производится квантование механической модели Лиувилля, с помощью матрицы Мёллера строятся операторы $\hat{p}$ и $e^{ \pm \gamma \hat{q}}$, вычисляются матричные элементы вершинных операторов частицы и описывается их реализация в терминах операторов нулевых мод $\hat{p}, \hat{q}$. Кроме того, с использованием свойств $S$-матрицы исследуется самосопряженное действие вершинных операторов на гильбертовом пространстве $L^{2}\left(\mathbb{R}_{+}\right)$ и, в частности, с помощью операторных методов выводится лиувиллевская амплитуда отражения.

3.1. Матрица Мёллера и операторы асимптотических нулевых мод. Для квантования канонического отображения плоскости $(P, Q)$ на полуплоскость $(p, q)(24)$ введем матрицу Мёллера [15]

$$
\widehat{U}=\lim _{\tau \rightarrow-\infty} \widehat{U}(\tau),
$$

которая задается асимптотиками унитарного оператора

$$
\widehat{U}(\tau)=e^{\frac{i}{\hbar} \widehat{H} \tau} e^{-\frac{i}{\hbar} \hat{H}_{0} \tau}
$$

Операторы, соответствуюшие двум фазовым пространствам, связаны тогда соотношениями

$$
\hat{p}=\widehat{U} \widehat{P} \widehat{U}^{+}, \quad e^{ \pm \gamma \hat{q}}=\widehat{U} e^{ \pm \gamma \widehat{Q}} \widehat{U}^{+} .
$$

Для упрощения последуюших вычислений удобно использовать обозначения

$$
x=\gamma Q-\ln \frac{\alpha}{m}, \quad \alpha=\frac{\hbar \gamma^{2}}{4 \pi} .
$$

Запишем лиувиллевский и свободньй гамильтонианы в $x$-представлении:

$$
\widehat{H}=\hbar \alpha\left(-\partial_{x}^{2}+e^{2 x}\right), \quad \widehat{H}_{0}=-\hbar \alpha \partial_{x}^{2} .
$$

Эти операторы действуют в гильбертовом пространстве $L^{2}(\mathbb{R})$. Собственные состояния гамильтониана $\widehat{H}$ суть функшии Кельвина [3], рассмотренные в приложении Б. В "бра-кет"-записи эти собственные состояния будут обозначаться как $\left|\Psi_{k}\right\rangle$, а для собственных состояний гамильтониана $\widehat{H}_{0}$ будем использовать обозначения $|k\rangle,-\infty<k<$ $+\infty$. Собственные состояния в обоих случаях имеют одинаковую нормировку $\left\langle k \mid k^{\prime}\right\rangle=$ $\delta\left(k-k^{\prime}\right)=\left\langle\Psi_{k} \mid \Psi_{k^{\prime}}\right\rangle$ и одинаковые собственные значения $E=\hbar \alpha k^{2}\left(=p^{2} /(4 \pi)\right)$. Здесь, однако, мы должны подчеркнуть, что спектр гамильтониана $\widehat{H}_{0}$ является вырожденным $(|k\rangle \neq|-k\rangle)$, в то время как спектр гамильтониана $\widehat{H}$ невырожденный $\left(\left|\Psi_{k}\right\rangle=\left|\Psi_{-k}\right\rangle\right)$; система $\left|\Psi_{k}\right\rangle$ является полной уже при $k>0$ (см. (П.10)). Это характеризует ситуацию для полуплоскости в квантово-механическом случае; в классическом случае она была рассмотрена в п. 2.3 . 
Для экспоненциального оператора (29) мы имеем, таким образом, "смешанное" спектральное разложение

$$
\widehat{U}(\tau)=\int_{0}^{+\infty} d k \int_{-\infty}^{+\infty} d k^{\prime}\left|\Psi_{k}\right\rangle U\left(k, k^{\prime} ; \tau\right)\left\langle k^{\prime}\right|
$$

в котором $U\left(k, k^{\prime} ; \tau\right)=e^{i \alpha\left(k^{2}-k^{2}\right) \tau}\left\langle\Psi_{k} \mid k^{\prime}\right\rangle$. В приложении Б показано, что матричньй элемент $\left\langle\Psi_{k} \mid k^{\prime}\right\rangle$ является расходящимся и должен рассматриваться как обобщенная функция. Его регуляризация $\left\langle\Psi_{k} \mid k^{\prime}\right\rangle_{\varepsilon}$ дается формулой (П.19).

С учетом (П.19) и известных формул теории рассеяния

$$
\lim _{t \rightarrow-\infty} \frac{e^{i\left(E-E^{\prime}\right) t}}{E-E^{\prime} \pm i \varepsilon}=-2 \pi i \theta( \pm \epsilon) \delta\left(E-E^{\prime}\right), \quad E=\alpha k^{2}
$$

регуляризованное ядро разложения (33) имеет предел

$$
\lim _{\tau \rightarrow-\infty} U_{\varepsilon}\left(k, k^{\prime} ; \tau\right)=a_{k} \theta\left(k^{\prime}\right) \delta\left(k-k^{\prime}\right) .
$$

Согласно (П.11)-(П.14) $a_{k}$ есть коэффициент “движущейся влево” волны собственной функции $\Psi_{k}(x)$ при $x \rightarrow-\infty \mathrm{c}\left|a_{k}\right|=1$, и поэтому $\left|\Psi_{k}^{-}\right\rangle=a_{k}\left|\Psi_{k}\right\rangle$ может быть названа собственным in-состоянием гамильтониана $\widehat{H}[16]$. Тогда матрица Мёллера принимает вид

$$
\widehat{U}=\int_{0}^{+\infty} d k\left|\Psi_{k}^{-}\right\rangle\langle k|
$$

где при интегрировании учитывается невырожденный спектр гамильтониана $\widehat{H}$ с $k>0$. Оператор, эрмитово сопряженный к матрице Мёллера, получается с помощью аналогичных вычислений:

$$
\widehat{U}^{+}=\lim _{\tau \rightarrow-\infty} e^{i \widehat{H}_{0} \tau / \hbar} e^{-i \widehat{H} \tau / \hbar}=\int_{0}^{+\infty} d k|k\rangle\left\langle\Psi_{k}^{-}\right| .
$$

Важно отметить, что оператор $\widehat{U}(\tau)$ является унитарным при конечных $\tau$, но в пределе $\tau \rightarrow-\infty$ сохраняется только одно их двух условий унитарности

$$
\widehat{U} \widehat{U}^{+}=\hat{I}, \quad \widehat{U}^{+} \widehat{U}=\int_{0}^{+\infty} d k|k\rangle\langle k|
$$

Эта неунитарность является следствием различия спектров операторов $\widehat{P}$ и $\hat{p}$.

Преобразование (30) формально сохраняет эрмитовость, и из (36), (37) следует спектральное разложение для оператора $\hat{p}$ на полупрямой:

$$
\hat{p}=\hbar \gamma \int_{0}^{+\infty} d k\left|\Psi_{k}^{-}\right\rangle k\left\langle\Psi_{k}^{-}\right|
$$


Оператор импульса $\hat{p}$, таким образом, самосопряжен, $\left|\Psi_{k}^{-}\right\rangle$- его собственное состояние с собственным значением $p=\hbar \gamma k>0$, и, снова используя (36), (37), мы получаем полезные соотношения:

$$
\hat{p} \widehat{U}=\widehat{U} \widehat{P}, \quad \widehat{U}^{+} \hat{p}=\widehat{P} \widehat{U}^{+} .
$$

В $x$-представлении операторы $\widehat{P}$ и $e^{ \pm \gamma \widehat{Q}}$ имеют вид

$$
\widehat{P}=-i \hbar \gamma \partial_{x}, \quad e^{-\gamma \widehat{Q}}=\frac{m}{\alpha} e^{-x}, \quad e^{\gamma \widehat{Q}}=\frac{\alpha}{m} e^{x}
$$

и удовлетворяют коммутационным соотношениям $\left[\widehat{P}, \hat{e}^{ \pm \gamma \widehat{Q}}\right]=\mp i \gamma \hbar e^{ \pm \gamma \widehat{Q}}$. Умножая эти соотношения слева и справа соответственно на $\widehat{U}$ и $\widehat{U}^{+}$, из (40) получаем согласованный с каноническим квантованием результат:

$$
\left[\hat{p}, \hat{e}^{ \pm \gamma \hat{q}}\right]=\mp i \gamma \hbar e^{ \pm \gamma \hat{q}}
$$

Можно также вычислить матричные элементы $\left\langle\Psi_{k}^{-}\left|e^{ \pm \gamma \hat{q}}\right| \Psi_{k^{\prime}}^{-}\right\rangle$. Используя формулы $(30),(36),(37),(41)$ и ортонормированность собственных состояний $\left|\Psi_{k}^{-}\right\rangle$, имеем

$$
\left\langle\Psi_{k}^{-}\left|e^{ \pm \gamma \hat{q}}\right| \Psi_{k^{\prime}}^{-}\right\rangle=\left(\frac{\alpha}{m}\right)^{ \pm 1}\left\langle k\left|e^{ \pm \hat{x}}\right| k^{\prime}\right\rangle
$$

Матричные элементы в правой части последнего выражения могут быть получены с помощью собственных состояний $|k\rangle$ в $x$-представлении, $\sqrt{2 \pi}\langle x \mid k\rangle=e^{i k x}$, в виде

$$
\left\langle k\left|e^{ \pm \hat{x}}\right| k^{\prime}\right\rangle=\frac{1}{2 \pi} \int_{-\infty}^{+\infty} d x e^{-i\left(k-k^{\prime} \pm i\right) x}=\delta\left(k-k^{\prime} \pm i\right) .
$$

$\delta$-Функция комплексного аргумента определяется на классе аналитических пробных функций стандартным образом [17], и мы будем использовать равенство

$$
\int_{0}^{+\infty} d k^{\prime} \delta\left(k-k^{\prime} \pm i\right) \psi\left(k^{\prime}\right)=\psi(k \pm i)
$$

Поскольку $\left|\Psi_{k}^{-}\right\rangle$- собственное состояние оператора $\hat{p}$, вернемся к первоначальным переменным нулевых мод и отождествим $\sqrt{\hbar \gamma}|p\rangle=\left|\Psi_{k}^{-}\right\rangle$для $p=\hbar \gamma k$. Собственные состояния $|p\rangle$ нормированы условием $\left\langle p \mid p^{\prime}\right\rangle=\delta\left(p-p^{\prime}\right)$, и они определяют $p$-представление волновыми функциями $\psi(p)=\langle p \mid \Psi\rangle \in L^{2}\left(\mathbb{R}_{+}\right)$, которое связывает $\psi(p)$ с волновыми функциями $Q$-представления $\Psi(x)$ :

$$
\psi(p)=\int_{-\infty}^{+\infty} d x \Psi_{k}^{*}(x) \Psi(x) \text { при } p=\hbar \gamma k
$$


Это преобразование является унитарным, поскольку система собственных состояний $\Psi_{k}(x)$ полна. Значит, $\hat{p} \psi(p)=p \psi(p)$, и операторы $e^{ \pm \gamma \hat{q}}$ в силу $(43),(44)$ действуют следующим образом:

$$
e^{-\gamma \hat{q}} \psi(p)=\frac{m}{\alpha} \psi(p-i \hbar \gamma), \quad e^{\gamma \hat{q}} \psi(p)=\frac{\alpha}{m} \psi(p+i \hbar \gamma)
$$

Эти соотношения определяют действие операторов нулевых мод в гильбертовом пространстве $L^{2}\left(\mathbb{R}_{+}\right)$. Однако необходимо еще рассмотреть свойства эрмитовости экспоненциальных $q$-операторов, что будет подробно сделано в п. 3.3.

Отметим, что любой оператор $F(p)$, который задается спектральным разложением, подобным (39):

$$
F(\hat{p})=\int_{0}^{+\infty} d p|p\rangle F(p)\langle p|,
$$

удовлетворяет соотношениям, аналогичным $(40), \quad F(\hat{p}) \widehat{U}=\widehat{U} F(\widehat{P})$ или $\widehat{U}^{+} F(\hat{p})=$ $F(\widehat{P}) \widehat{U}^{+}$, и, следовательно, для такого оператора справедливо следуюшее правило умножения:

$$
F(\hat{p}) e^{ \pm \gamma \hat{q}}=e^{ \pm \gamma \hat{q}} F(\hat{p} \mp i \hbar \gamma)
$$

Последнее соотношение было использовано для алгебраического построения лиувиллевских вершинных операторов в работах [4], [6].

3.2. Вершинные операторы и их матричные элементы. Уравнения Гейзенберга, полученные на основе гамильтониана $\widehat{H}=\hbar \alpha\left(-\partial_{x x}^{2}+e^{2 x}\right)$, дают операторное уравнение $\ddot{\hat{x}}(\tau)+4 \alpha^{2} e^{2 \hat{x}(\tau)}=0$, которое имеет подобную теории поля Лиувилля классическую форму (7). Однако вершинный оператор частишы

$$
\widehat{V}(\tau)=e^{-\gamma \widehat{Q}(\tau)}=e^{i \widehat{H} \tau / \hbar} e^{-\gamma \widehat{Q}} e^{-i \widehat{H} \tau / \hbar}
$$

удовлетворяет уравнению

$$
\frac{1}{2}(\ddot{\widehat{V}}(\tau) \widehat{V}(\tau)+\widehat{V}(\tau) \ddot{\widehat{V}}(\tau))-\dot{\hat{V}}(\tau)^{2}+2 \alpha^{2} \widehat{V}(\tau)^{2}=4 m^{2}
$$

деформированному по сравнению с соответствующим классическим уравнением

$$
\ddot{V}(\tau) V(\tau)-\dot{V}(\tau)^{2}=4 m^{2}
$$

Мы наблюдаем связанное с правилом упорядочения изменение для члена $\ddot{V}(\tau) V(\tau)$ и квантовую поправку, пропорциональную $\alpha^{2}$. Даже квантовая модификация калибровочно-инвариантного уравнения (12) содержит квантовую деформацию:

$$
\ddot{\widehat{V}}(\tau)=\frac{\gamma^{2}}{2 \pi}(\widehat{H} \widehat{V}(\tau)+\widehat{V}(\tau) \widehat{H})+\alpha^{2} \widehat{V}(\tau)
$$


Здесь снова можно провести параллель с теорией Лиувилля, в которой (анти)киральные операторы удовлетворяют квантово-механически деформированным уравнениям Шредингера [5], [6].

Можно вычислить матричные элементы вершинных операторов механической модели (подчеркнем, что мы также используем обозначение $V$ для $V_{-1}$ )

$$
V_{2 b}\left(k, k^{\prime} ; \tau\right)=\left\langle\Psi_{k}^{-}\left|e^{2 b \gamma \hat{Q}(\tau)}\right| \Psi_{k^{\prime}}^{-}\right\rangle
$$

С учетом (31) и (П.15) равенство (53) принимает вид

$$
V_{2 b}\left(k, k^{\prime} ; \tau\right)=\left(\frac{\alpha}{m}\right)^{2 b} d_{k}^{*} d_{k^{\prime}} e^{i \alpha\left(k^{2}-k^{\prime 2}\right) \tau} \int_{-\infty}^{\infty} d x K_{i k}\left(e^{x}\right) e^{2 b x} K_{i k^{\prime}}\left(e^{x}\right)
$$

Однако интеграл в последнем выражении хорошо определен только при $b>0$ и расходится при $b \leqslant 0$. Выражение (54) определяет, таким образом, обобшенную функцию, которая является ядром вершинного оператора, и для того чтобы вычислить это ядро, нам необходимо его продолжение с положительных $b$ на отрицательные. При подстановке (П.9) в (54) и интегрировании по переменной $u=e^{x}$ интеграл разлагается в произведение двух интегралов типа (П.18), что после введения обозначений

$$
\kappa=\frac{k+k^{\prime}}{2}, \quad \rho=\frac{k-k^{\prime}}{2}
$$

дает для $b>0$ результат [9]

$$
V_{2 b}=\left(\frac{\alpha}{m}\right)^{2 b} e^{4 i \alpha \rho \kappa \tau} 4^{b-i \rho} \frac{\Gamma(b+i \kappa) \Gamma(b-i \kappa)}{\Gamma(i \rho+i \kappa) \Gamma(i \rho-i \kappa)} \frac{\Gamma(b+i \rho) \Gamma(b-i \rho)}{4 \pi \Gamma(2 b)} .
$$

Чтобы получить вершинную функцию для отрицательных $b$, необходимо продолжить (56) как обобшенную функцию, что не было сделано ранее. Поскольку $\kappa>|\rho|$, неопределенности могут возникнуть в точках $b=-n, n=0,1,2, \ldots$ Вблизи этих точек при $b=-n+\epsilon$ выражение (56) ведет себя как

$$
\frac{1}{\pi} \frac{\epsilon}{\left(k-k^{\prime}\right)^{2}+\epsilon^{2}}
$$

В приложении В показано, что эта обобщенная функция на классе голоморфных пробных функций имеет следуюшее продолжение из области положительных в область отрицательных значений $\epsilon$ :

$$
\frac{1}{\pi} \frac{\epsilon}{\left(k-k^{\prime}\right)^{2}+\epsilon^{2}}+\delta\left(k-k^{\prime}+i \epsilon\right)+\delta\left(k-k^{\prime}-i \epsilon\right) .
$$


Таким образом, продолжение выражения (56) на отрицательные значения $b$ порождает пару $\delta$-функций комплексного аргумента всякий раз, когда $b$ проходит через отрицательное целое значение, и для $b=-|b|$ имеем следующий результат:

$$
\begin{aligned}
V_{-2|b|}= & V_{-2|b|}^{+}(\kappa, \rho ; \tau)+\left(\frac{m}{\alpha}\right)^{2|b|} \sum_{l=0}^{[|b|]} C_{2|b|}^{l} e^{-4 \alpha(|b|-l) \kappa \tau} \times \\
& \times \frac{\Gamma(-|b|+i \kappa) \Gamma(-|b|-i \kappa)}{4^{l} \Gamma(-|b|+l+i \kappa) \Gamma(-|b|+l-i \kappa)} \delta[2 \rho-2 i(|b|-l)]+ \\
& +C_{2|b|}^{l} e^{4 \alpha(|b|-l) \kappa \tau} \frac{\Gamma(-|b|+i \kappa) \Gamma(-|b|-i \kappa)}{4^{2|b|-l} \Gamma(|b|-l+i \kappa) \Gamma(|b|-l-i \kappa)} \delta[2 \rho+2 i(|b|-l)] .
\end{aligned}
$$

Здесь $V_{-2|b|}^{+}(\kappa, \rho ; \tau)$ определяется правой частью выражения $(56)$, в котором $b$ заменено на $-|b|,[|b|]$ - целая часть $|b|$ и

$$
C_{2|b|}^{l}=\prod_{j=0}^{l-1} \frac{2|b|-j}{j+1} .
$$

В частности, функция $V_{-2|b|}^{+}$обращается в нуль при полуцелых значениях $|b|$ благодаря полюсу $\Gamma(-2|b|)$ и дает члены, пропорциональные $\delta(\rho)$ при целых $|b|$, так что при $2|b|=n$ мы имеем

$$
V_{-n}(\kappa, \rho ; \tau)=\left(\frac{m}{\alpha}\right)^{n} \sum_{l=0}^{n} C_{n}^{l} e^{-2(n-2 l) \kappa \tau} \prod_{j=0}^{l-1} \frac{1}{4 \kappa^{2}+(n-2 j)^{2}} \delta[2 \rho-i(n-2 l)],
$$

где $C_{n}^{l}$ - биномиальные коэффициенты.

Рассмотрим матричный элемент вершинного оператора $\widehat{V}(\tau)$, который соответствует случаю $n=1$ в (61):

$$
V\left(k, k^{\prime} ; \tau\right)=\frac{m}{\alpha}\left(e^{-\alpha\left(k+k^{\prime}\right) \tau} \delta\left(k-k^{\prime}-i\right)+\frac{e^{\alpha\left(k+k^{\prime}\right) \tau}}{4 k k^{\prime}} \delta\left(k-k^{\prime}+i\right)\right) .
$$

Из этого выражения и из (47) мы можем легко вывести структуру вершинного оператора частицы в виде

$$
\widehat{V}(\tau)=e^{-\gamma \hat{p} \tau /(4 \pi)} e^{-\gamma \hat{q}} e^{-\gamma \hat{p} \tau /(4 \pi)}+\omega^{2} \frac{e^{\gamma \hat{p} \tau /(4 \pi)}}{\hat{p}} e^{\gamma \hat{q}} \frac{e^{\gamma \hat{p} \tau /(4 \pi)}}{\hat{p}} .
$$

Здесь наблюдается упорядочение, аналогичное упорядочению в выражении для редуцированного квантового лиувиллевского оператора (26), и с помощью (49) нетрудно проверить, что оператор (63) действительно удовлетворяет обоим операторным уравнениям (51) и (52).

Можно показать, что вершинный оператор $\widehat{V}_{-n}$ является $n$-й степенью оператора (63), однако для положительных или нецелых отрицательных значений $2 b$ оператор $\widehat{V}_{2 b}$, полученный из (56), или первый член в (59), соответственно, дается (при использовании фурье-преобразований) бесконечным рядом $q$-экспонент, почти так же, как в квантовой теории поля Лиувилля [6]. 
3.3. Самосопряженность операторов на полуплоскости. Необходимо задаться вопросом, являются ли вершинные операторы $\widehat{V}(\tau)(63)$ и $\hat{\mathbf{V}}(\tau)$ (26) физическими операторами, действие которых в гильбертовом пространстве $L^{2}\left(\mathbb{R}_{+}\right)$эрмитово.

Оператор (63) при $\tau=0, e^{-\widehat{Q}}$, в $x$-представлении, очевидно, является самосопряженным, и поэтому оператор $\widehat{V}(\tau)=e^{-\widehat{Q}(\tau)}$ также является самосопряженным для любого $\tau$. Но эта самосопряженность должна быть совместима с временно́й эволюцией. Для вычисления зависящих от времени матричных элементов

$$
\langle\psi|\widehat{V}(\tau)| \psi\rangle=\left\langle\psi(\tau)\left|e^{-\widehat{Q}}\right| \psi(\tau)\right\rangle
$$

состояние $|\psi(\tau)\rangle$ должно лежать в той же области, где определен оператор $e^{-\widehat{Q}}$, независимо от $\tau$. Но в $x$-представлении это не очевидно. Поэтому мы будем использовать $p$-представление, в котором временна́я эволюция становится простой.

Поскольку асимптотически при $\tau \rightarrow \pm \infty$ остается только один из вершинных членов, кажется привлекательным потребовать эрмитовость для каждого из членов в (63) по отдельности. Однако доказательство эрмитовости для $e^{\gamma \hat{q}}$ потребовало бы весьма специальных граничных условий на голоморфные функции $\psi(p)$ на полуплоскости при $\operatorname{Re} p=0$. Эта интересная сама по себе математическая задача до сих пор не решена.

Однако можно ожидать, что вершинньй оператор (63), ввиду его симметрии относительно преобразований, задаваемых $S$-матрищей $\widehat{S}=\widehat{\mathcal{P}} S(p)$ [18], становится самосопряженным как целое.

Для модели частицы $S(p)$ является мультипликативной амплитудой отражения (П.16) с $p=\hbar \gamma k$, а $\widehat{\mathcal{P}}$ - оператор четности, $\widehat{\mathcal{P}} \psi(p)=\psi(-p) . \quad S$-матрица $\widehat{S}$ заменяет $i n$-приходяший первый член в $(63), e^{-\gamma \widehat{Q}_{\text {in }}(\tau)}=e^{-\gamma(\hat{q}+\hat{p} \tau /(2 \pi))}$, на out-уходяший второй член, $e^{-\gamma \widehat{Q}_{\text {out }}(\tau)}=\omega^{2}[\hat{p}(\hat{p}+i \hbar \gamma)]^{-1} e^{\gamma(\hat{q}+\hat{p} \tau /(2 \pi))}$,

$$
e^{-\gamma \widehat{Q}_{\text {in }}(\tau)} \widehat{S}=\widehat{S} e^{-\gamma \widehat{Q}_{\text {out }}(\tau)},
$$

и наоборот, так что вершинный оператор частицы остается инвариантным, $\widehat{S} \widehat{V}(\tau) \widehat{S}^{-1}=$

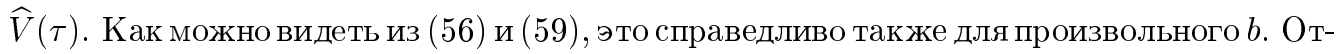
метим, что $S$-матричное преобразование есть просто квантовый вариант преобразования симметрии (18). Оператор $\widehat{S}$ также отображает гильбертово пространство $i n$-полей $L^{2}\left(\mathbb{R}_{+}\right)$на все пространство $L^{2}\left(\mathbb{R}_{-}\right)$out-полей, для волновых функций $\psi(p) \in L^{2}\left(\mathbb{R}_{+}\right)$, $\tilde{\psi}(p) \in L^{2}\left(\mathbb{R}_{-}\right)$это отображение задается соотношением $\tilde{\psi}(-p)=S(p) \psi(p)$. Последнее соотношение определяется формулой (46) с использованием (П.15), (П.16) и $\Psi_{-k}^{*}(x)=$ $d_{-k}^{*} K_{-i k} e^{x}=S_{k} \Psi_{k}^{*}(x)$.

Благодаря этим свойствам мы можем распространить определение матричного элемента вершинного оператора с гильбертова пространства на полуплоскости $L^{2}\left(\mathbb{R}_{+}\right)$на гильбертово пространство на плоскости $L^{2}(\mathbb{R})$ :

$$
\int_{0}^{\infty} d p \psi_{+}^{*}(p) \widehat{V}(\tau) \psi_{+}(p)=\frac{1}{2} \int_{-\infty}^{\infty} d p \Psi^{*}(p) \widehat{V}(\tau) \Psi(p) .
$$


Здесь $\Psi(p)=\psi(p)$ при $p>0, \Psi(p)=\tilde{\psi}(p)=S(-p) \psi(-p)$ при $p<0$ и $\Psi(p) \in L^{2}(\mathbb{R})$ удовлетворяет условию $\widehat{S} \Psi(p)=\Psi(p)$. Самосопряженность оператора (63), очевидно, имеет место на $L^{2}(\mathbb{R})$ для голоморфных волновых функций $\Psi(p)$. Как следствие самосопряженность $\widehat{V}(\tau)$ на $L^{2}\left(\mathbb{R}_{+}\right)$требует наличия для функции $\psi(p)$ такого голоморфного расширения на отрицательную полупрямую, что $\psi(-p)=\tilde{\psi}(-p)=S(p) \psi(p)$, которое действительно является инвариантным относительно временно́й эволюции $\psi(p, \tau)=$ $e^{i p^{2} \tau /(4 \pi \hbar)} \psi(p)$. Такие функции даются выражением $\psi(p)=d^{*}(p) f(p)$, где $d(p)$ определено в (П.15) с $p=\hbar \gamma k$ и $f(p)$ - четная голоморфная функция, $f(-p)=f(p)$. Интеграл (65) хорошо определен в сингулярной точке $p=0$ оператора $\widehat{V}(\tau)$, поскольку $\psi(0)=0$ вследствие $\left.d(p)\right|_{p=0}=0$.

Можно, наконец, задаться вопросом, является ли лиувиллевский вершинный оператор в вакуумном секторе $\widehat{\mathbf{V}}(\tau)(26)$ инвариантным относительно $S$-матричного преобразования $\widehat{S}_{\mathrm{L}}=\widehat{\mathcal{P}} S_{\mathrm{L}}(p)$, которое меняет $i n$-приходящую часть нулевых мод на деформированную out-уходящую часть подобно (64). Мы исследовали получающееся в результате уравнение инвариантности

$$
\widehat{S}_{\mathrm{L}}^{-1} \widehat{\mathbf{V}}(\tau) \widehat{S}_{\mathrm{L}}=\widehat{\mathbf{V}}(\tau)
$$

и нашли для $S_{\mathrm{L}}(p)$ амплитуду отражения, предложенную в [12] (где $\left.b^{2}=2 \alpha, 4 \pi b P=\gamma p\right)$,

$$
S_{\mathrm{L}}(p)=-\left(m_{\alpha}^{2} \Gamma^{2}(2 \alpha)\right)^{-i p /(\hbar \gamma)} \frac{\Gamma(i \gamma p /(4 \pi \alpha))}{\Gamma(-i \gamma p /(4 \pi \alpha))} \frac{\Gamma(i \gamma p /(2 \pi))}{\Gamma(-i \gamma p /(2 \pi))}
$$

Остановимся на этом более подробно. $S$-матрица теории поля Лиувилля $\widehat{\mathbf{S}}$ связывает in- и out-поля лиувиллевского вершинного оператора

$$
e^{-\gamma \hat{\varphi}(\tau, \sigma)}=e^{-\gamma \hat{\varphi}_{\text {in }}(\tau, \sigma)}+e^{-\gamma \hat{\varphi}_{\text {out }}(\tau, \sigma)},
$$

где $\hat{\varphi}_{\text {in }}(\tau, \sigma)$ - оператор свободного поля $(3), \hat{\phi}(\tau, \sigma)=\hat{\phi}(z)+\hat{\bar{\phi}}(\bar{z})$, и согласно приложению А

$$
e^{-\gamma \hat{\varphi}_{\text {out }}(\tau, \sigma)}=m_{\alpha}^{2}: e^{-\gamma \hat{\phi}(\tau, \sigma)} \int_{0}^{2 \pi} \int_{0}^{2 \pi} d y d \bar{y} G_{\alpha}(z, \bar{z} ; y, \bar{y}) e^{2 \gamma[\hat{\phi}(y)+\hat{\phi}(\bar{y})]} .
$$

Таким образом, как и в случае частицы, получаем

$$
e^{-\gamma \hat{\varphi}_{\text {in }}(\tau, \sigma)} \widehat{\mathbf{S}}=\widehat{\mathbf{S}} e^{-\gamma \hat{\varphi}_{\text {out }}(\tau, \sigma)} .
$$

Амплитуда отражения теории поля Лиувилля $S_{\mathrm{L}}(p)$ определена в работе [12] выражением

$$
\widehat{\mathbf{S}}|p, \mathbf{0}\rangle=S_{\mathrm{L}}(p)|-p, \mathbf{0}\rangle,
$$


где $|p, \mathbf{0}\rangle$ - $p$-зависимое вакуумное состояние. Взяв матричный элемент от операторного тождества (70) между вакуумными состояниями $|p, \mathbf{0}\rangle$ и $\left\langle p^{\prime}, \mathbf{0}\right|$ и используя явные in / out-выражения (26), из коэффициентов при $\delta\left(p^{\prime}+p-i \hbar \gamma\right)$ выводим соотношение

$$
S_{\mathrm{L}}(p)=\omega_{\alpha}^{2} \frac{1}{p(p-i \hbar \gamma)} \frac{\Gamma\left(-i \gamma p /(2 \pi)-\hbar \gamma^{2} /(2 \pi)\right)}{\Gamma\left(i \gamma p /(2 \pi)+\hbar \gamma^{2} /(2 \pi)\right)} \frac{\Gamma(i \gamma p /(2 \pi))}{\Gamma(-i \gamma p /(2 \pi))} S_{\mathrm{L}}(p-i \hbar \gamma) .
$$

Это уравнение для $S_{\mathrm{L}}(p)$ эквивалентно (66), и его решение может быть записано в виде

$$
S_{\mathrm{L}}(p)=\frac{\Gamma(i \gamma p /(2 \pi))}{\Gamma(-i \gamma p /(2 \pi))} S_{\alpha}(p),
$$

где $S_{\alpha}(p)$ удовлетворяет более простому уравнению (без гамма-функций)

$$
S_{\alpha}(p)=\omega_{\alpha}^{2} \frac{1}{p(p-i \hbar \gamma)} S_{\alpha}(p-i \hbar \gamma)
$$

Это просто уравнение для амплитуды отражения в механической модели $S(p)$ (П.16), в котором $\omega$ заменяется на входящий в $(26)$ деформированный параметр $\omega_{\alpha}$. Решение этого уравнения дает для лиувиллевской амплитуды отражения $S_{\mathrm{L}}(p)$ результат $(67)$ и впервые убедительно доказывает его.

Этот результат дает надеж ду на более глубокое осмысление основанных на континуальном интегрировании гипотез относительно лиувиллевских трехточечных функций с помошью операторного подхода [18], [19]. Для доказательства самосопряженности вершинных операторов лиувиллевских полей требуется полная $S$-матрица. Операторное уравнение для лиувиллевской $S$-матрицы и его решение будет рассмотрено в другой работе.

\section{4. ЗАКЛЮЧЕНИЕ}

Мы детально исследовали динамику лиувиллевской частищы, которая описьвает теорию поля Лиувилля в секторе нулевых мод, проквантовали нулевые моды на полуплоскости и доказали, что соответствующие операторы являются самосопряженными вследствие скрытых симметрий вершинных операторов относительно $S$-матричного преобразования. Для того чтобы получить матричные элементы вершинных операторов частищы, был разработан метод продолжения обобщенных функций по параметру взаимодействия.

Мы научились работать с квантовой теорией поля Лиувилля в секторе нулевых мод исходя из динамики лиувиллевской частицы, в качестве интересного результата вычислили амплитуду отражения с помощью операторного метода и использовали эту амплитуду для доказательства самосопряженности соответствующих вершинных операторов.

Можно надеяться, что обнаруженные аналитические свойства матричных элементов для частицы окажутся полезными для установления связей с результатами, полученными альтернативно операторным методом и в рамках подхода континуального интегрирования, что позволит проверить предполагаемый вид лиувиллевских трехточечных функций. 
ПРИЛОЖЕНИЕ А

\section{Редуцированный вершинный оператор}

Возьмем операторную форму лиувиллевской вершинной функции (2) из [6] (в наших обозначениях), вычислим нормально упорядоченный осцилляторный матричный элемент (25) при $\lambda=-\gamma$ :

$$
\widehat{\mathbf{V}}(\tau)=: e^{-\gamma(\hat{q}+\hat{p} \tau /(2 \pi))}\left(1+m_{\alpha}^{2} \int_{0}^{2 \pi} \int_{0}^{2 \pi} d y d \bar{y} G_{\alpha}(z, \bar{z} ; y, \bar{y}) e^{2 \gamma(\hat{q}+\hat{p}(y+\bar{y}) /(4 \pi))}\right):
$$

и используем правило упорядочения

$$
: e^{2 a q} A(p):=e^{a \hat{q}} A(\hat{p}) e^{a \hat{q}}
$$

Индекс $\alpha$ символизирует квантовые деформации,

$$
m_{\alpha}^{2}=\frac{\sin \pi \alpha}{\pi \alpha} m^{2}, \quad \alpha=\frac{\hbar \gamma^{2}}{4 \pi},
$$

$G_{\alpha}(z, \bar{z} ; y, \bar{y})$ - функция Грина (5), (6), деформированная следуюшим образом:

$$
\operatorname{sh}^{2} \frac{\gamma p}{2} \rightarrow \operatorname{sh}^{2}\left(\frac{\gamma p}{2}\right)+\sin ^{2}\left(\frac{\hbar \gamma^{2}}{4}\right)
$$

и умноженная на фактор малых расстояний $f_{\alpha}(z-y) f_{\alpha}(\bar{z}-\bar{y})$, даваемый выражением

$$
f_{\alpha}(x)=\left(4 \sin ^{2} \frac{x}{2}\right)^{\alpha}
$$

Здесь следует отметить, что факторы малых расстояний являются следствием конформной инвариантности, а деформаций (П.3) и (П.4) возникают в результате локальности. С учетом соотношения [20]

$$
\int_{0}^{2 \pi} d x\left(4 \sin ^{2} \frac{x}{2}\right)^{\alpha} e^{\gamma p x /(2 \pi)}=\frac{2 \pi \Gamma(1+2 \alpha) e^{\gamma p / 2}}{\Gamma(1+\alpha+i \gamma p /(2 \pi)) \Gamma(1+\alpha-i \gamma p /(2 \pi))}
$$

и тождества

$$
\begin{gathered}
\Gamma\left(1-\alpha-i \frac{\gamma p}{2 \pi}\right) \Gamma\left(1-\alpha+i \frac{\gamma p}{2 \pi}\right) \Gamma\left(1+\alpha-i \frac{\gamma p}{2 \pi}\right) \Gamma\left(1+\alpha+i \frac{\gamma p}{2 \pi}\right)= \\
=\frac{(\pi \alpha)^{2}+(\gamma p / 2)^{2}}{\operatorname{sh}^{2}(\gamma p / 2)+\sin ^{2} \pi \alpha}
\end{gathered}
$$

мы приходим к выражению $(26)$, где $\omega_{\alpha}$ содержит все $p$-независимые вклады и имеет классический предел $2 \pi m / \gamma$. 
ПРИЛОЖЕНИЕ Б

\section{Собственные состояния механического лиувиллевского гамильтониана}

Решения стационарного уравнения Шредингера

$$
-\Psi_{k}^{\prime \prime}(x)+e^{2 x} \Psi_{k}(x)=k^{2} \Psi_{k}(x)
$$

даются функциями Кельвина (модифиццрованными функциями Бесселя) $\Psi_{k}(x)=$ $c_{k} K_{i k}\left(e^{x}\right)$ [3] (см. также формулы (2.16)-(2.22) в [9]), где $c_{k}$ - нормировочный коэффициент. Функции Кельвина являются вешественными, $K_{i k}^{*}(u)=K_{-i k}(u)=K_{i k}(u)$, и обладают полезным интегральным представлением [20]

$$
K_{i k}(u)=\frac{1}{2} \int_{-\infty}^{+\infty} d y e^{-u \operatorname{ch} y} e^{i k y}
$$

Собственные состояния $\Psi_{k}(x)$ быстро стремятся к нулю при $x \rightarrow \infty$ и осциллируют при $x \rightarrow-\infty$. Спектр $E=\hbar \alpha k^{2}$ является невырожденным, но точка с нулевой энергией $k=0$ должна быть исключена из спектра, поскольку соответствующая волновая функция расходится при $x \rightarrow-\infty$, и для собственных состояний $\Psi_{k}(x)$ мы берем только значения $k>0$.

Условия нормировки и полноты

$$
\int_{-\infty}^{+\infty} d x \Psi_{k}(x) \Psi_{k^{\prime}}(x)=\delta\left(k-k^{\prime}\right), \quad \int_{0}^{+\infty} d k \Psi_{k}(x) \Psi_{k}\left(x^{\prime}\right)=\delta\left(x-x^{\prime}\right)
$$

справедливы [21], если $c_{k}=\sqrt{2 k \operatorname{sh} \pi k} / \pi$. Из теории рассеяния известно, что решения стационарного уравнения Шредингера дают асимптотически полную информацию о процессе рассеяния [16]. Асимптотики $K_{\nu}(u)$ при $u \rightarrow 0$ [20]

$$
K_{\nu}(u) \rightarrow-\frac{\pi}{2}\left[\frac{2^{-\nu} u^{\nu}}{\sin (\pi \nu) \Gamma(1+\nu)}-\frac{2^{\nu} u^{-\nu}}{\sin (\pi \nu) \Gamma(1-\nu)}\right]
$$

дают для волновых функций

$$
\Psi_{k}(x) \rightarrow a_{k}^{*} \frac{e^{i k x}}{\sqrt{2 \pi}}+a_{k} \frac{e^{-i k x}}{\sqrt{2 \pi}} \text { при } x \rightarrow-\infty .
$$

Коэффициент out-уходяшей волны

$$
a_{k}=-i \frac{2^{i k}}{\Gamma(1-i k)} \sqrt{\frac{\pi k}{\operatorname{sh} \pi k}}
$$


является фазой вследствие соотношения

$$
\Gamma(1+i k) \Gamma(1-i k)=\frac{\pi k}{\operatorname{sh} \pi k}
$$

Поэтому собственные состояния $\Psi_{k}^{-}(x)=a_{k} \Psi_{k}(x)$ имеют асимптотики

$$
\Psi_{k}^{-}(x) \rightarrow \frac{e^{i k x}}{\sqrt{2 \pi}}+S_{k} \frac{e^{-i k x}}{\sqrt{2 \pi}} \text { при } x \rightarrow-\infty
$$

Они, очевидно, удовлетворяют условиям нормировки и полноты (П.10) и даются выражением

$$
\Psi_{k}^{-}(x)=d_{k} K_{i k}\left(e^{x}\right), \quad d_{k}=\sqrt{\frac{2}{\pi}} \frac{2^{i k}}{\Gamma(-i k)} .
$$

С учетом (П.13) мы легко находим амплитуду отражения [9]

$$
S_{k}=2^{2 i k} \frac{\Gamma(i k)}{\Gamma(-i k)}=\frac{d_{k}}{d_{k}^{*}}
$$

Импульсное представление для собственных состояний определяется преобразованием Фурье от функции Кельвина

$$
\left\langle\Psi_{k} \mid k^{\prime}\right\rangle=\frac{1}{\sqrt{2 \pi}} \int_{-\infty}^{+\infty} d x e^{i k^{\prime} x} \Psi_{k}(x)
$$

Этот интеграл является расходящимся и должен рассматриваться как обобшенная функция. Регуляризуем (П.17) с помошью множителя $e^{\epsilon x}$ и, используя (П.9), а также интеграл [20]

$$
\int_{-\infty}^{+\infty} d y \frac{e^{i k y}}{\operatorname{ch}^{\mu} y}=\frac{2^{\mu-1}}{\Gamma(\mu)} \Gamma\left(\frac{\mu+i k}{2}\right) \Gamma\left(\frac{\mu-i k}{2}\right), \quad \operatorname{Re} \mu>0
$$

получим регуляризованное импульсное представление собственного состояния $\Psi_{k}(x)[10]$

$$
\left\langle\Psi_{k} \mid k^{\prime}\right\rangle_{\epsilon}=c_{k} \frac{2^{\epsilon+i k^{\prime}}}{4 \sqrt{2 \pi}} \Gamma\left(\frac{\epsilon+i k^{\prime}-i k}{2}\right) \Gamma\left(\frac{\epsilon+i k^{\prime}+i k}{2}\right)
$$

которое используется в (35). 
ПРИЛОЖКЕНИЕ В

\section{Продолжение интегральных операторов}

Поскольку свойства лиувиллевской механической модели и динамики свободной частицы на полупрямой $x<0$ похожи, достаточно описать использованную в п. 3.2 процедуру аналитического продолжения только для случая свободной частицы. С учетом требуемых граничных условий $\psi(0)=0$ гамильтониан $\widehat{H}=-\hbar^{2} \partial_{x x}^{2}$ имеет собственные состояния $\psi_{k}(x)=\sqrt{2 / \pi} \sin k x$ с собственными значениями $\hbar^{2} k^{2}$. Такой спектр является невырожденным, как и в случае динамики лиувиллевской частицы, и мы выбираем $k>0$.

Вычисление матричных элементов экспонент от оператора координаты

$$
V_{\beta}\left(k, k^{\prime}\right)=\left\langle\psi_{k}\left|e^{\beta \hat{x}}\right| \psi_{k^{\prime}}\right\rangle=\frac{2}{\pi} \int_{-\infty}^{0} d x e^{\beta x} \sin k x \sin k^{\prime} x
$$

таким образом упрошается по сравнению с формулой (54). Оператор $e^{\beta \hat{x}}$ ограничен при $\beta>0$, и мы имеем

$$
V_{\beta}\left(k, k^{\prime}\right)=\frac{1}{\pi}\left(\frac{\beta}{\left(k-k^{\prime}\right)^{2}+\beta^{2}}-\frac{\beta}{\left(k+k^{\prime}\right)^{2}+\beta^{2}}\right) .
$$

Однако при $\beta<0$ интеграл (П.20) расходится и $V_{\beta}\left(k, k^{\prime}\right)$ снова должна рассматриваться как обобщенная функция, которая является ядром оператора $e^{\beta \hat{x}}$ в $k$-представлении. Таким образом, нам необходимо продолжение (П.21) по $\beta$, действующее на пробные функции $\psi(k)$. Это продолжение для произвольной функции $\psi(k)$ не существует. Поскольку $k$ и $k^{\prime}$ положительны, второй член в (П.21) регулярен для любого $\beta$ и его продолжение тривиально, в то время как "наивное" продолжение первого члена сингулярно при $\beta=0$. Действительно, интеграл

$$
I_{\beta}(\psi ; k)=\frac{1}{\pi} \int_{0}^{\infty} d k^{\prime} \frac{\beta}{\left(k^{\prime}-k\right)^{2}+\beta^{2}} \psi\left(k^{\prime}\right)
$$

имеет скачок при $\beta=0$,

$$
\lim _{\beta \rightarrow \pm 0} I_{\beta}(\psi ; k)= \pm \psi(k) .
$$

Чтобы построить продолжение интеграла (П.22), разложим его в сумму двух интегралов $I_{\beta}=I_{\beta}^{(+)}+I_{\beta}^{(-)}$

$$
I_{\beta}^{( \pm)}(\psi ; k)= \pm \frac{1}{2 \pi i} \int_{0}^{\infty} d k^{\prime} \frac{\psi\left(k^{\prime}\right)}{k^{\prime}-(k \pm i \beta)},
$$

и выберем класс пробных функций $\psi(k)$, которые голоморфны на полуплоскости $\operatorname{Re} k>0$ и стремятся к нулю при $\operatorname{Re} k \rightarrow \infty$. Введем интегралы Коши

$$
J_{\beta}^{( \pm)}(\psi ; k)= \pm \frac{1}{2 \pi i} \oint d z \frac{\psi(z)}{z-(k \pm i \beta)},
$$

в которых интегрирование ведется по контурам, показанным на рис. 3 . 


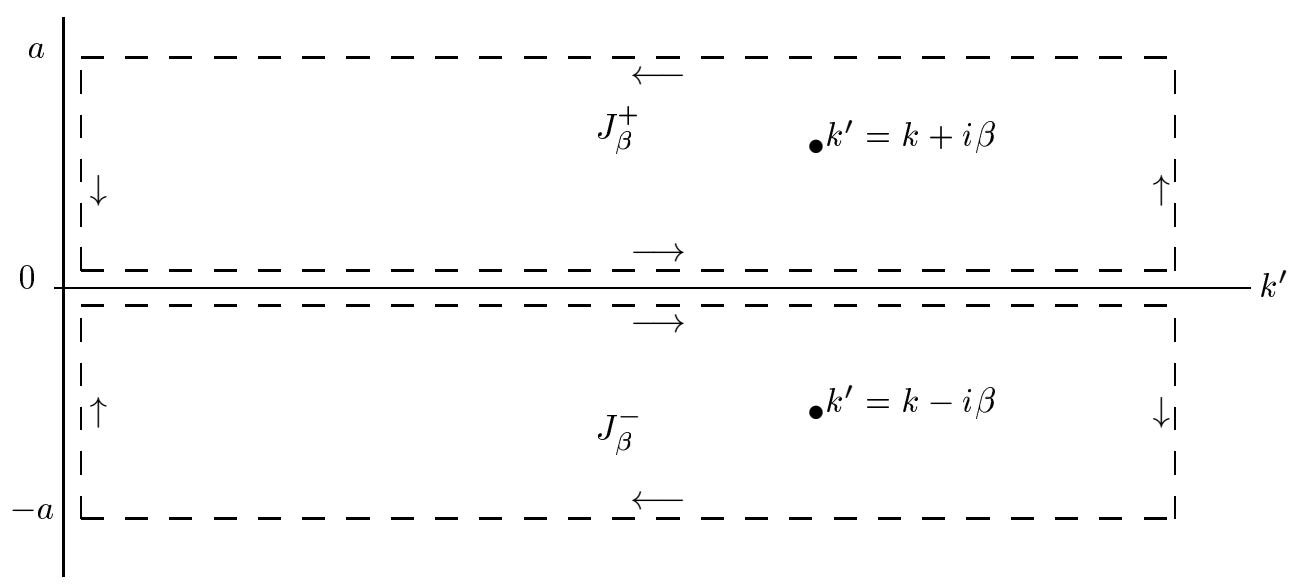

Рис. 3

Интегралы Коши хорошо определены при $a>\beta>0$, где $a$ - параметр, характеризуюший контуры. В этом случае подынтегральные выражения в (П.23) имеют полюсы в точках $z=k \pm i \beta$, и $J_{\beta}^{( \pm)}=\psi(k \pm i \beta)$. Тогда $I_{\beta}^{( \pm)}$дается выражением

$$
\begin{aligned}
I_{\beta}^{( \pm)}(\psi ; k)= & \pm \frac{1}{2 \pi} \int_{0}^{a} d \eta \frac{\psi(i \eta)}{-k \pm i(\eta-\beta)} \pm \\
& \pm \frac{1}{2 \pi i} \int_{0}^{\infty} d \xi \frac{\psi(\xi \pm i a)}{\xi-k \pm i(a-\beta)}+\psi(k \pm i \beta) .
\end{aligned}
$$

В таком виде $I_{\beta}^{( \pm)}(\psi ; k)$, очевидно, допускает гладкое продолжение из области положительных $(0<\beta<a)$ в область отрицательных $\beta$. Обозначим продолжение (П.24) при $\beta=-a$ через $\tilde{I}_{-a}^{( \pm)}(\psi ; k)$. Поскольку при $\beta=-a$ контурные интегралы (П.23) обрашаются в нуль, первые два члена в $\tilde{I}_{-a}^{( \pm)}$совпадают с $I_{-a}^{( \pm)}$, и мы имеем

$$
\tilde{I}_{-a}^{( \pm)}(\psi ; k)=I_{-a}^{( \pm)}(\psi ; k)+\psi(k \mp i a) .
$$

Таким образом, мы имеем следующий набор интегральных операторов $\tilde{I}_{\beta}$ :

$$
\tilde{I}_{\beta}(\psi ; k)=\left\{\begin{array}{lr}
I_{\beta}(\psi ; k), & \beta>0, \\
\psi(k), & \beta=0, \\
I_{\beta}(\psi ; k)+\psi(k+i \beta)+\psi(k-i \beta), & \beta<0 .
\end{array}\right.
$$

Поэтому ядро (П.21) для отрицательных $\beta$ принимает вид

$$
V_{\beta}\left(k, k^{\prime}\right)=\frac{1}{\pi}\left(\frac{\beta}{\left(k-k^{\prime}\right)^{2}+\beta^{2}}-\frac{\beta}{\left(k+k^{\prime}\right)^{2}+\beta^{2}}\right)+\delta\left(k-k^{\prime}+i \beta\right)+\delta\left(k-k^{\prime}-i \beta\right) .
$$

Этим определяется продолжение, которое используется в п. 3.2 для вычисления матричных элементов вершинных операторов в модели лиувиллевской частишы. 
Благодарности. Мы благодарны Т. Куртрайту, Д. Фаирли, С. Торну и С. Закосу, которые привлекли наше внимание к своим работам по динамике лиувиллевской частицы, и С. Форду, М. Ройтеру, В. Рюэлю, Г. Саввиди, Р. Сейлеру и Дж. Тешнеру за обсуждения. Г. Джорджадзе благодарен DESY Zeuthen за гостеприимство. Работа была поддержана грантами DFG, INTAS, РФФИ (грант № 02-01-00484) и GAS.

\section{Список литературы}

[1] J. Liouville. J. Math. Pures Appl. 1853. V. 18. P. 71.

[2] Г. П. Джсорджадзе. ТМФ. 1979. Т. 41. № 1. С. 33.

[3] E. D'Hoker, R. Jackiw. Phys. Rev. D. 1982. V. 26. P. 3517.

[4] E. Braaten, T. Curtright, C. Thorn. Ann. Phys. 1983. V. 147. P. 365.

[5] J.-L. Gervais, A. Neveu. Nucl. Phys. B. 1984. V. 238. P. 125; P. 398; J. Schnittger. TM 1995. T. 104. № 1. C. 158.

[6] H. J. Otto, G. Weigt. Z. Phys. C. 1986. V. 31. P. 219; G. Weigt. Canonical quantisation of the Liouville theory, quantum group structures, and correlation functions. In: Pathways to Fundamental Theories. Singapore: World Scientific, 1993. P. 227; hep-th/9208075.

[7] G. Jorjadze, G. Weigt. Nucl. Phys. B. 2001. V. 619. P. 232.

[8] Г. Вайгт, Г. Джсрджадзе, К. Форд. ТМФ. 2001. Т. 128. № 2. С. 249.

[9] E. Braaten, T. Curtright, G. Ghandour, C. Thorn. Ann. Phys. 1984. V. 153. P. 147.

[10] T. Curtright, D. Fairlie, C. Zachos. Phys. Rev. D. 1998. V. 58. P. 025002.

[11] I. Tsutsui, L. Feher. Progr. Theor. Phys. Suppl. 1995. V. 118. P. 173; T. Fülöp. J. Math. Phys. 1996. V. 37. P. 1617.

[12] A. B. Zamolodchikov, Al. B. Zamolodchikov. Nucl. Phys. B. 1996. V. 477. P. 577.

[13] H. Dorn, H. J. Otto. Phys. Lett. B. 1992. V. 291. P. 39; Nucl. Phys. B. 1994. V. 429. P. 375.

[14] J. Balog, L. Feher, P. Forgacs, L. O'Raifeartaigh, A. Wipf. Phys. Lett. B. 1989. V. 227. P. 214; Ann. Phys. 1992. V. 203. P. 269.

[15] C. Møller. Kgl. Danske Vidensk. Selsk. Mat.-Fys. Medd. 1945. V. 23. № 1.

[16] С. Швебер. Введение в релятивистскую квантовую теорию поля. М.: ИЛ, 1963.

[17] И. М. Гельфанд, Г. Е. Шилов. Обобщенные функции и действия над ними. М.: ГИФМЛ, 1959.

[18] J. Teschner. Class. Q. Grav. 2001. V. 18. P. 153.

[19] C. B. Thorn. Phys. Rev. D. 2002. V. 66. P. 027702.

[20] И. С. Градитейн, И. М. Рыжик. Таблицы интегралов, сумм, рядов и произведений. М.: Физматгиз, 1963.

[21] Н. Н. Лебедев. ДАН СССР. 1946. Т. 52. № 8. С. 561; Г. Бейтмен, А. Эрдейи. Таблицы интегральных преобразований. Т. 1, 2. М.: Наука, 1969, 1970.

Поступила в редакцию 7.V.2003 г. 